\title{
Bestrahlung der Brust erhöht Herzinfarkt-Risiko
}

\author{
Die Radiotherapie des Mamma- \\ karzinoms führt zu Kollateral- \\ schäden am Herzen. In einer \\ Fall-Kontroll-Studie wurde das \\ Ausmaß des Risikos quantifiziert.
}

$V^{2}$ on 2.168 Brustkrebs-Patientinnen, die zwischen 1958 und 2001 in Schweden oder Dänemark bestrahlt wurden, war bei 963 nach der Therapie ein schweres Koronarereignis eingetreten. Die Strahlenbelastung des Herzens wurde anhand von Dosis und Bestrahlungsfeld errechnet. Die mittlere Gesamtdosis betrug demnach 4,9 Gy, bei Bestrahlung der linken Brust naturgemäß deutlich mehr (6,6 vs. 2,9 Gy).

Das Risiko für ein gravierendes Koronarereignis (Herzinfarkt, koronare Revaskularisierung oder KHK-bedingter Tod) stieg linear mit der mittleren auf das Herz applizierten Strahlendosis mit jedem Gray um 7,4\%. Einen unteren Schwellenwert scheint es nicht zu geben, so Sarah C. Darby von der Universitätsklinik Oxford und Kollegen.
Das erhöhte Koronarrisiko machte sich schon innerhalb der ersten fünf Jahre bemerkbar (16,3\% mehr Ereignisse) und blieb mindestens 20 Jahre lang erhalten $(8,2 \%$ mehr Ereignisse). Frauen mit kardialen Risikofaktoren hatten erwartungsgemäß mehr koronare Komplikationen, aber eine Radiotherapie erhöhte das Risiko bei ihnen in gleichem Maße wie bei Frauen ohne Risikofaktoren.

Kombiniert man die Ergebnisse mit westeuropäischen Daten zur Inzidenz von Koronarereignissen, lässt sich das absolute Risiko für eine strahleninduzierte KHK abschätzen: So steigert etwa eine Radiotherapie, bei der die mittlere Dosis am Herzen 3 Gy beträgt, bei einer 50-jährigen nicht prädisponierten Frau das Risiko für einen vorzeitigen kardialen Tod von 1,9 auf 2,4\%, das für ein akutes Koronarereignis von 4,5 auf 5,4\%. Dieselbe Bestrahlung bewirkt bei einer 50-Jährigen mit mindestens einem kardialen Risikofaktor eine Zunahme des Sterberisikos von 3,4 auf 4,1\%. Auch moderne Radiotherapie-Regimes errei-

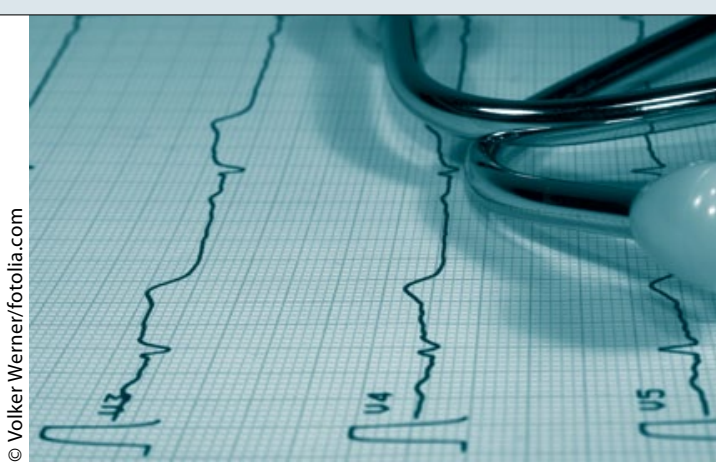

chen übrigens bei den meisten Patientinnen noch zwischen 1 und 5 Gy.

Fazit: Die Bestrahlung eines Mammakarzinoms steigert das Risiko für ein schweres Koronarereignis linear mit der Strahlenbelastung und über viele Jahre hinweg. Nach Einschätzung von Javid Moslehi von der Harvard Medical School in Boston könnte das „nur die Spitze des Eisbergs darstellen", denn eine Strahlentherapie begünstigt auch andere kardiale Erkrankungen, die gar nicht berücksichtigt wurden (Perikarderkrankungen, Arrhythmien). Und auch Anthrazykline und HER2-Antikörper wirken kardiotoxisch. Beate Schumacher

Darby SC et al. Risk of ischemic heart disease in women after radiotherapy for breast cancer. N Engl J Med. 2013;368(11):987-98.

\section{Biomarker helfen bei Therapieentscheidung}

\section{Bereits die Zwischenergebnisse der Chemo-NO-Studie legten nahe, dass die Testung des Biomarkers Plasminogen-Aktivator vom Urokinase-Typ (UPA) und seines Inhibitors PAI-1 bei Frauen mit nodal-negativem Brustkrebs von prognostischem Nutzen ist. Jetzt liegen die 10-Jahres-Daten dazu vor.}

D ie Chemo-N0-Studie, an der fast 650 Patientinnen mit nodal-negativem Brustkrebs teilnahmen, ist die erste prospektive Studie zum Nutzen dieser Biomarker über einen so langen Zeitraum (im Median 113 Monate) in dieser Indikation.

Die aktuelle Analyse zeigt, dass Patientinnen mit niedrigen Werten (uPA $\leq$ $3 \mathrm{ng} / \mathrm{mg}$; PAI-1 $\leq 14 \mathrm{ng} / \mathrm{mg}$ ) eine sehr gute Prognose haben. Nach zehn Jahren lebten $87 \%$ der Frauen krankheitsfrei, die Gesamtüberlebensrate lag bei fast $90 \%$ - ohne jede adjuvante systemische Therapie. Patientinnen mit hohen Werten (uPA > $3 \mathrm{ng} / \mathrm{mg}$; PAI-1 > $14 \mathrm{ng} / \mathrm{mg}$ ) hatten eine 10-Jahres-Rezidivrate von
$23 \%$, bei niedrigen Werten betrug sie nur 12,9\% (Hazard Ratio [HR] 1,84, 95\%-Konfidenzintervall $1,1-3,0 ; \mathrm{p}=$ 0,017).

Patientinnen mit hohen Werten profitieren von einer adjuvanten Therapie mit CMF (Cyclophosphamid, Methotrexat und 5-Fluorouracil): Im Vergleich zu alleiniger Beobachtung ließ sich die Rezidivrate dadurch signifikant verringern (32,1 vs. $21,3 \%$; HR 0,48; $\mathrm{p}=0,019$ ).

Die Erkenntnisse der Studie haben sich inzwischen auch in der AGO-Leitlinie von 2012 niedergeschlagen: „Bei niedriger uPA/PAI-1-Konzentration kann insbesondere Patientinnen mit nach traditionellen Kriterien unklarem
Rezidivrisiko (G2) eine adjuvante Chemotherapie erspart werden." (Empfehlungsgrad 0, Level of Evidence 1a)

Die Ergebnisse gelten wohl nur für HER2-negative Patientinnen, da HER2positive Patientinnen inzwischen von der Behandlung mit Trastuzumab profitieren. In der Studie war der HER2-Status noch nicht erhoben worden.

Fazit: Die abschließende 10-Jahres-Analyse der Chemo-N0-Studie bestätigt den prognostischen Nutzen des uPA/PAI-1Tests bei Frauen mit nodal-negativem Brustkrebs. Etwa der Hälfte davon kann aufgrund der guten Prognose bei niedrigen Testwerten eine adjuvante Chemotherapie erspart werden. Peter Leiner

Harbeck $\mathrm{N}$ et al. Ten-year analysis of the prospective multicentre Chemo-NO trial validates American Society of Clinical Oncology (ASCO)recommended biomarkers $\mathrm{UPA}$ and $\mathrm{PAI}-1$ for therapy decision making in node-negative breast cancer patients. Eur J Cancer. 2013;49(8):1825-35. 